\title{
CSCI/RCPSC HENRY FRIESEN LECTURE The Past and the Future of Neurogenetics
}

\author{
Guy Rouleau MD PhD FRCPC \\ Inge Meijer PhD
}

Centre of excellence in Neuromics, Université de Montréal and Hôpital St-Justine Montréal, Québec.

Presented 27th September, 2007 at RCPSC meeting, Winnipeg

Dr. Rouleau was the 2007 Henry Friesen Lecturer

Clin Invest Med 2007; 30 (6): E269-E273.
"To wrest from nature the secrets which have perplexed philosophers in all ages, to track to their sources the cause of disease ... these are our ambitions"

Sir William Osler

The main aim in neurogenetics is to characterize and understand the genetic causes underlying neurological diseases. Over time, progress has been made in several aspects of neurogenetics. In fact, the evolution of neurogenetics largely resembles the steps currently undertaken when executing a neurogenetics study. These steps include identification of a disease in a family, clinical description and characterization of the family, genetic analysis, and finally understanding the function of the causative gene. Along those lines, the evolution of neurogenetics could be divided in four eras namely the descriptive, the medical technological, the molecular genetics and the personalized medicine era.

\section{Descriptive era, 1800 -}

Historically, neurogenetics started when neurologists noticed that certain diseases segregated in families. These families were closely followed and the observations were described in the literature. Neurologists in different parts of the world contributed to this era and a few examples are discussed below.

In France, a key person was Jean Marie Charcot (1825-1893), who can be considered one of the founders of modern neurology. ${ }^{1}$ Although not the initial person to describe cases with amyotrophic lateral sclerosis (ALS), Charcot further characterized the disorder by linking the motor neuron loss and corticospinal degeneration to the clinical signs and by naming the disease ALS. ${ }^{2,3}$ In one report, Charcot describes ALS in two affected brothers. It is important to note that all these observations were made during a time when the motor system was still poorly understood. In his busy career Charcot described many other medical conditions including Charcot's disease and a group of diseases called Charcot-Marie-Tooth disease. A few years after Charcot's description of ALS, William Osler also reported an American family that clearly had an autosomal dominant familial form of ALS. ${ }^{4}$ Interestingly, the causative mutation, Ala4Val, was identified decades later and is the most common recurrent mutation in the world causing a rapidly progressive form of ALS.

In Germany, Ernst Adolf Gustav Gottfried von Strümpell (1853-1925) described hereditary diseases 
including Strümpell-Lorrain disease and Wilson's disease.

Finally, John Hughlings Jackson (1835-1911) in London, England contributed to the description of epilepsy and neuro-opthalmological diseases. ${ }^{5} \mathrm{He}$ is most famous for his work on epilepsy which included observations that he made of his wife Elizabeth Dade Jackson. The commonly known clinical entities named after him are Jackson's epilepsy and the Jacksonian march.

Aside from the advances in descriptive neurology there were important observations in the field now known as genetics. Therefore, the work of the Australian monk Gregor Mendel (1822-1884) should also be underlined. He used common pea plants (Pisum sativum) to elucidate the basic principles in genetics; namely the principle of segregation and the principle of independent assortment. His work was published in a monograph entitled Experiments with Plant Hybrids. The principles proved to be inherent to the understanding of disease segregation in families according to the different modes of inheritance.

\section{The medical technological era, 1900 -}

It is a general principle that progress is driven by technology. The progress in neurogenetics was no different. In fact, the technological advances made since the 50s were key in the rapid progress of neurogenetics. Modern neurology is heavily dependent on noninvasive imaging as well as electromyography (EMG) and nerve conduction. Developed in the 1950s, EMG and nerve conduction studies enabled neurologists to distinguish between neuropathies and myopathies. Before the EMG, physicians relied on the patient history, physical examination, tissue biopsy, and postmortem pathologic correlation to diagnose neuromuscular disorders. For example, Peter Dyck and colleagues categorized the hereditary motor and sensory neuropathies in HSMN type 1 (the demyelinating form) and type 2 (the axonal form) with the aid of EMG. ${ }^{6}$
The invention of computed tomography by GN Hounsfield in 1972 was a major development in medicine, in particular in neurology where it allows for a view inside the cranium. A few years later Ernst and Edelstein (Nobel Prize 1992) developed magnetic resonance imaging (MRI), which allowed greater definition of the brain, particularly in diseases of the posterior fossa and the spinal cord. One can now exactly determine the extent of cerebellar atrophy or leukodystrophy, for example. This type of imaging is also very useful in determining the location of demyelinating plaques in multiple sclerosis in the brain and the spinal cord. In fact, Hereditary Spastic Paraplegia can only be diagnosed after an MRI has excluded demyelinating disease or other forms of spinal cord disease.

The medical technological era mainly contributed to improved diagnosis and phenotype definition. In genetic studies, a clear diagnosis is crucial to the analysis. In addition, a better defined phenotype allows for sub-grouping (endo-phenotyping) of phenotypes for association studies.

\section{The molecular era 1953 -}

During the molecular era the underlying genetic causes of the previously described diseases finally started to be identified. This era represents an era with exponential progress in neurogenetics. A few important highlights are mentioned in Table 1.

The invention of the polymerase chain reaction (PCR) was the most important development that eventually permitted other technological advances such as

TABLE 1. The major developments in molecular genetics

\begin{tabular}{c|l}
\hline 1953 & $\begin{array}{l}\text { Structure of DNA deduced (Watson, Crick, Wilk- } \\
\text { ins, Franklin) }\end{array}$ \\
\hline 1968 & DNA code deciphered, DNA sequencing \\
\hline 1978 & First germline-engineered mammals (mice) \\
\hline 1983 & PCR \\
\hline 1988 & Launch of Human Genome Project \\
\hline 1991 & First somatic gene transfer experiments begun \\
\hline 2000 & "Working draft" of the human genome sequence \\
\hline 2003 & Human Genome sequence "complete" \\
\hline $2005-2007$ & CNV, CGH, SNP chips \\
\hline
\end{tabular}


improved sequencing, the genome project, haplotype mapping etc. ${ }^{7} \mathrm{PCR}$ is a mainstay in any genetics laboratory and it increased the speed as well as the ease of DNA analysis tremendously in all fields including neurogenetics. The rapid development of technology has led to sequencing of the entire human genome together with the annotation of the genes it contains. PCR as well as the human genome project enabled faster identification of disease causing mutations.

There is no consensus on how many genes there are in the genome but estimates average around 30,000 genes which could be producing up to 400,000 different proteins. So far, $\sim 2,000$ genes are known to cause a disease ( $7 \%$ ) (OMIM). Even though it is not known how many mutated genes are embryonic lethal or have no effect at all or how many single genes cause several diseases, it is clear that with only $7 \%$ of genes characterized, many more disease genes will be discovered in the future. Furthermore, a recent study showed that the genetic load of a new loss of function (LOF) allele is $\mathrm{U}=0.02$ per generation per gamete. Assuming 25,000 genes, this would be equal to 106,000 new LOF alleles per year worldwide. ${ }^{8}$ Assuming there are $133,000,000$ births per year and a generation time of 25 years, the human genome is completely saturated annually worldwide with severe loss-of-function mutations. In other words, every possible LOF mutation has occurred and exists in the population unless it is embryonic lethal or has been selected against in evolution. Therefore, clinicians should continue identifying and describing new disorders since many more genes and associated phenotypes have yet to be discovered. In addition, technological advances have permitted improved diagnosis since it was previously only based on clinical data but can now be complemented with imaging plus genetic results. To illustrate this further, two motor neuron diseases, Hereditary Spastic Paraplegia (HSP) and Amyotrophic Lateral Sclerosis, will be discussed.

HSP is a disorder characterized by upper motor neuron degeneration that leads to progressive lower limb spasticity. ${ }^{9}$ Since the year 2000, 10 genes and 14 novel loci have been identified, bringing the total of HSP loci to 36. The clinical presentation is generally quite similar for each locus; it is only via genetics that the subtypes can be identified. The 14 currently known HSP causing genes have a wide range of function and a common pathophysiological pathway has not yet been established. The leading hypothesis is that the disease is caused by a defect in axonal maintenance as well as in molecular trafficking, which has also been associated with other neurodegenerative diseases. In the future, drugs could be developed to act on specific disease pathways and a diagnostic test could guide the choice of treatment.

ALS is also an upper and lower motor neuron disease which leads to death usually within 3-5 years after diagnosis. In ALS there are less genes identified, partly because large families are rare. ${ }^{10}$ One of these genes, SOD1, was identified as the cause of $15-20 \%$ of all familial ALS cases. However, it only explains 1-2\% of all ALS cases, as most cases of ALS are sporadic. Many studies on the pathophysiology of SOD1 have been reported in the last year. With the development of an antibody specific to mutant SOD ${ }^{11}$ as well as the discovery that oxidized SOD1 results in an ALS phenotype in mice ${ }^{12}$, it can be hypothesized that non-familial ALS can be caused by modifications of the SOD1 protein. These latest developments open many new avenues for treatments and possibly prevention. In addition, if this hypothesis turns out to be supported, an SOD1 based treatment would have a large impact.

\section{The future of neurogenetics 2007 -}

The rapid development of new technology mainly improves the speed at which different tests can be done. Several high-throughput techniques have become widely used in recent years such as single nucleotide polymorphism (SNP) chips and comparative genome hybridization (CGH). These two techniques allow for an increased coverage of the human genome and the detection of copy number variants (small deletions 
and duplications). In turn, this signifies that more causative genes will be identified and characterized by molecular biology in the near future. In addition, methods are developed to improve our understanding of the pathophysiology. One such approach is the interactome study. A recent study demonstrated that a common pathway of 54 proteins is involved in the pathogenesis of 23 inherited ataxias and these can serve as potential targets in therapeutic avenues. ${ }^{13}$

Another example of high-throughput pathophysiologically based studies that will become more common in the future is the S2D (Synapse to Disease) project in our laboratory. The project has as an aim to identify and characterize genes involved in common developmental brain diseases. This approach is based on a large scale sequencing project of 1,000 synaptic genes in selected affected individuals in the hope of identifying de novo mutations.

Both of these projects have as an ultimate goal to facilitate pathway specific drug development. Another area in which neurogenetics will play an important role is in pharmacogenetics and the development of drugs specific to certain genotypes. This will enable personalized medicine in which each treatment will be decided according to a patient's genotype. Furthermore, the first diploid sequence of an individual, Craig Venter, was published this year. ${ }^{14}$ Although the technology is still too expensive and labour intensive, in the future this could become a standard analysis for each patient possibly in the form of a genome on a chip.

This type of personalized medicine will also require improved diagnostic screening and database management while adhering to the highest ethical standards. Unfortunately, there is no centralized Canadian diagnostic screening facility at a time when many diagnostic tests are being discovered in Canada and should be made available to the public.

The concept of personalized medicine will be illustrated in the following example:

Mr. X presents with progressive spastic paraparesis.

(C) 2007 CIM

Clin Invest Med • Vol 30, no 6, December 2007

E272
The neurologist determines a clinical diagnosis of HSP following a thorough examination and necessary imaging. Mr.X then undergoes whole genome sequencing through a certified diagnostic screening lab followed by selective examination of the HSP genes. The variants will be assessed using bioinformatic tools to confirm the causative mutation. Once the mutated gene is identified, the patient is notified by a medical geneticist who can, in conjunction with the neurologist, choose an appropriate drug for the involved pathway. The family members can also be tested and counseled with risk assessment and targeted prevention.

We have certainly come a long way since the days of Drs. Osler and Charcot. Many genetic causes of neurological diseases have since been identified and characterized. However, many more remain to be unraveled, even more so in complex diseases such as autism and mental retardation. The trend of exponential progress seen in the molecular era of neurogenetics will likely continue with the hope to develop better treatments for neurological diseases based on the understanding of the pathophysiology of neurogenetic diseases in the next coming years.

\section{References}

1. Tan S Y, Shigaki D, Charcot J-M (1825-1893): pathologist who shaped modern neurology. Singapore Med J 2007;48:383-4.

2. Charcot J-M, Joffroy A. Deux cas d'atrophie musculaire progressive avec lésions de la substance grise et des faiseaux antérolatéraux de la moelle épinière." Archives de physiologie normale et pathologique 1869; 2:354-67, 629-49, 744-60.

3. Rowland LP. How amyotrophic lateral sclerosis got its name: the clinical-pathologic genius of Jean-Martin Charcot. Arch Neurol 2001;58:512-5.

4. Osler W. On heredity in progressive muscular atrophy as illustrated in the Farr family of Vermont. Archives of Medicine 1880;4:316-20.

5. Swash M, Evans J. Hughlings Jackson's clinical research: evidence from contemporary documents. Neurology 2006;67:666-72.

6. Dyck PJ, Lambert EH. Lower motor and primary sensory neuron diseases with peroneal muscular atrophy. 
I. Neurologic, genetic, and electrophysiologic findings in hereditary polyneuropathies. Arch Neurol 1968;18:603-18.

7. Saiki RK, et al. Enzymatic amplification of betaglobin genomic sequences and restriction site analysis for diagnosis of sickle cell anemia. Science 1985;230:1350-4.

8. Kondrashov AS. Direct estimates of human per nucleotide mutation rates at 20 loci causing Mendelian diseases. Hum Mutat 2003;21:12-27.

9. Soderblom C, Blackstone C. Traffic accidents: molecular genetic insights into the pathogenesis of the hereditary spastic paraplegias. Pharmacol Ther 2006;109:42-56.

10.Pasinelli P, Brown RH. Molecular biology of amyotrophic lateral sclerosis: insights from genetics. Nat Rev Neurosci 2006;7:710-23.

11. Rakhit R, Robertson J, Vande Velde C, et al. An immunological epitope selective for pathological monomer-misfolded SOD1 in ALS. Nat Med 2007;13:754-9.

12.Urushitani M, Ezzi SA, Julien JP. Therapeutic effects of immunization with mutant superoxide dismutase in mice models of amyotrophic lateral sclerosis. Proc Natl Acad Sci U S A 2007;104:2495-50

13. Lim J, Hao T, Shaw C, et al. A protein-protein interaction network for human inherited ataxias and disorders of Purkinje cell degeneration. Cell 2006;125:801-14.

14.Levy S, Sutton G, Ng PC, et al. The Diploid Genome Sequence of an Individual Human. PLoS Biol 2007;5:e254. 